\title{
Watermarking Based on the Density Coefficients of Faber-Schauder Wavelets
}

\author{
Mohamed El hajji ${ }^{1}$, Hassin Douzi ${ }^{1}$, and Rachid Harba ${ }^{2}$ \\ ${ }^{1}$ RF-SIC, Faculté des sciences Ibn Zohr, Agadir, Maroc \\ ${ }^{2}$ PRISME Institut, Polytech'Orléans, 12 rue de Blois 45067 Orleans CEDEX 2
}

\begin{abstract}
The field of watermarking is of great development in recent years. There is still relevance to find high-performance methods that link robustness and discretion. In this paper, we will present a method of watermarking based on a mixed scale wavelet transformations of Faber-Schauder and the selection of high-density regions of the dominant coefficients. Simulation results demonstrate the robustness of our image watermarking procedure under various attacks and could serve for the image watermarking of identity and access cards.
\end{abstract}

Keywords: Digital watermarking, Faber-Schauder wavelet transformation, dominant coefficients.

\section{Introduction}

The digital watermarking tends to exploit the imperfections of the human visual system to embed invisible identification information into a digital content (video, audio or image), in order to protect it [10]. In the original document, we insert a signature (Watermark), which is formed by a sequence of bits. The insertion must respect the imperceptibility compared to the human visual system and the robustness (i.e. difficult to extract or to delete).

The wavelet transformation (DWT) is now widely used in image processing [12]. They are distinguished by a multi-scales decomposition of the image to separate the structures of different sizes. Most of the watermarking methods in the wavelet domain are working with scales separated [15]. For each scale, pixels are decorrelated by this transform and information is only present in a small number of wavelet coefficients: the dominant coefficients [6.7]. These coefficients are located mainly around the edge of the image and characterize all the information of the image. This is especially true for identities images which are the topic of our interest (the images scanned from sensitive documents: passports, identity cards, access cards....). The originality of our method is the use of mixed-scale visualization [6] ,that permit to distinguish very particular regions around the edges of the image, where we have a high density of dominant wavelet coefficients. These regions are characterized by the fact that they are highly textured regions, therefore ideal to hide information. It is also the regions with a high density of the dominant coefficients, therefore unavoidable in the characterization of the image and a priori stable for any transformation of the image. Another originality of our method is the use of the density of dominant coefficients as a criterion at the insertion and the extraction. 
In this paper, we present a method based on the density of wavelet coefficients. The Faber-Schauder wavelets are chosen since they are particularly well adapted for the watermarking [7].

After describing the mixed-scale visualization, we will propose a strategy for watermarking and test its robustness following various attacks.

\section{Faber-Schauder Wavelet Transform}

The algorithm of Faber-Schauder wavelet transform (FSWT) is simple to express by a lifting scheme $[6,16]$. We can consider a image as a sequence $f^{0}=\left(f_{m, n}^{0}\right)_{m, n \in \mathbb{Z}}$ of $L^{2}\left(\mathbb{Z}^{2}\right)$, Transform FSWT is done in three steps as shown in the following figure:

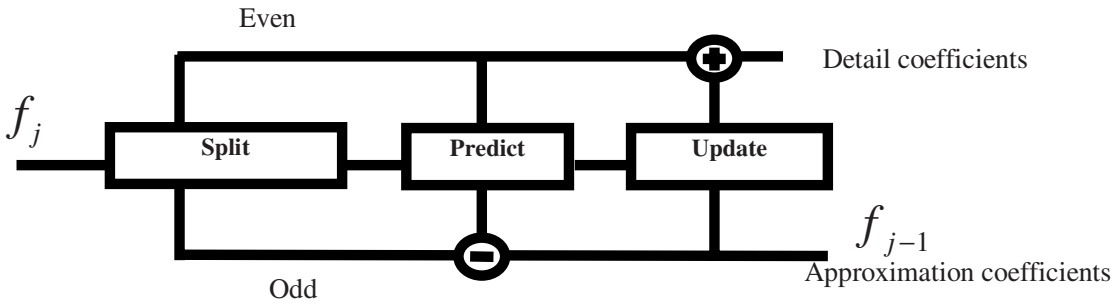

Fig. 1. The transformation by lifting scheme

The Scheme lifting of the FSWT [6] is given by the following algorithm:

$$
\text { TOFS : }\left\{\begin{array}{l}
\mathrm{f}_{\mathrm{ij}}^{0}=\mathrm{f}_{\mathrm{ij}} \quad \text { pour } \mathrm{i}, \mathrm{j} \in \mathbb{Z} \\
\text { pour } 1 \leq \mathrm{k} \leq \mathrm{N} \quad \text { et } \mathrm{i}, \mathrm{j} \in \mathbb{Z} \\
\mathrm{f}_{\mathrm{ij}}^{\mathrm{k}}=\mathrm{f}_{2 \mathrm{i}, 2 \mathrm{j}}^{\mathrm{k}-1} \\
\mathrm{~g}_{\mathrm{ij}}^{\mathrm{k} 1}=\left(\mathrm{g}_{\mathrm{ij}}^{\mathrm{k} 1}, \mathrm{~g}_{\mathrm{ij}}^{\mathrm{k} 2}, \mathrm{~g}_{\mathrm{ij}}^{\mathrm{k} 3}\right) \\
\mathrm{g}_{\mathrm{ij}}^{\mathrm{k} 1}=\mathrm{f}_{2 \mathrm{i}-1,2 \mathrm{j}}^{\mathrm{k}-1}-\frac{1}{2}\left(\mathrm{f}_{2 \mathrm{i}, 2 \mathrm{j}}^{\mathrm{k}-1}+\mathrm{f}_{2 \mathrm{i}+2,2 \mathrm{j}}^{\mathrm{k}-1}\right) \\
\mathrm{g}_{\mathrm{ij}}^{\mathrm{k} 2}=\mathrm{f}_{2 \mathrm{i}, 2 \mathrm{j}+1}^{\mathrm{k}-1}-\frac{1}{2}\left(\mathrm{f}_{2 \mathrm{i}, 2 \mathrm{j}}^{\mathrm{k}-1}+\mathrm{f}_{2 \mathrm{i}+2,2 \mathrm{j}+2}^{\mathrm{k}-1}\right) \\
\mathrm{g}_{\mathrm{ij}}^{\mathrm{k} 3}=\mathrm{f}_{2 \mathrm{i}+1,2 \mathrm{j}+1}^{\mathrm{k}-1}-\frac{1}{4}\left(\mathrm{f}_{2 \mathrm{i}, 2 \mathrm{j}}^{\mathrm{k}-1}+\mathrm{f}_{2 \mathrm{i}, 2 \mathrm{j}+2}^{\mathrm{k}-1}+\mathrm{f}_{2 \mathrm{i}+2,2 \mathrm{j}}^{\mathrm{k}-1}+\mathrm{f}_{2 \mathrm{i}+2,2 \mathrm{j}+2}^{\mathrm{k}-1}\right)
\end{array}\right.
$$

\section{The Watermarking by the Density of the Coefficients of Faber-Schauder Wavelet}

\subsection{Watermark Embedding}

The basic idea consists in inserting a mark on the edges detected by the density by block of coefficients of FSWT, while taking into account two important aspects: the robustness and invisibility. 
The algorithm for watermark embedding can be presented as following:

- Firstly we calculate FSWT of original image $\mathbf{I}$, to get a transformed image $\mathbf{F}$ in mixed scales representation [6].

- The second step consists of selecting the high density blocks of wavelet coefficients followed by a classification of these blocks in ascending order, and so to insert the mark in the important blocks (in the sense of density). The embedding can also be effected on all blocks selected. this way, the mark to embed may be redundant if it has a length less than the total number of blocks extracted.

- The third step consists to applying a spread spectrum method into the watermark $\mathbf{W}$ by using a random sequence broadband $\mathbf{U}$ [8], so, the gotten message reinforces the resistance of the signature to perturbations provoked on the image. Each block is going to carry information in related with a given bit of message obtained in the form of an additive term. The watermark embedding follows the following equation:

$$
C_{f}^{*}(i, j)=C_{f}(i, j)\left(1+\alpha W_{k}(i, j)\right)
$$

Here the constant $\boldsymbol{\alpha}$ gives the watermark strength.

$\mathrm{C}_{\mathrm{f}}^{*}(\mathrm{i}, \mathrm{j})$ : The marked coefficient (with $\mathrm{i}, \mathrm{j}=1$.. the block size).

$\mathrm{C}_{\mathrm{f}}(\mathrm{i}, \mathrm{j})$ : Initial coefficient of the selected block.

$W_{k}(i, j)$ : the sequence obtained by the spread spectrum method.

After the marking of a block, we replace it at the corresponding block in the image $\mathbf{F}$.

- Finally, we reconstruct the changed image by using the inverse wavelet transform.

The selected blocks are those containing large densities of dominant wavelet coefficients as shown in Fig. 2.

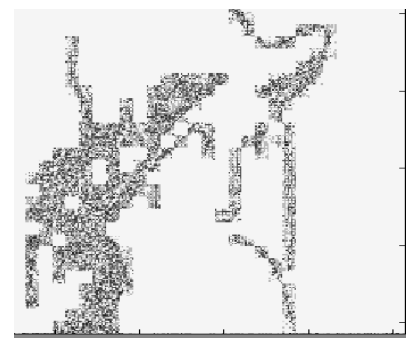

Fig. 2. The selection of blocks of high-density of the dominant coefficients (For Lena image: $8 \times 8$ blocks, $\mathrm{SC}=10, \mathrm{SD}=20 \%$ )

The selection of blocks is based on the following selection criterion:

- A threshold $S C$ is fixed to select the dominant coefficients: A coefficient is considered dominant if its absolute value is superior to $S C$.

- A threshold $S D$ of density is fixed to select insertion blocks: All block whose density passes this threshold will be selected as a dominant block. 


\subsection{Watermark Detection}

The insertion of the watermark is made by a spread spectrum in the dominant blocks. At the reception of a marked image, we calculate the correlation with the random sequence $\mathbf{U}$ used for the modulation [8].

More precisely we proceed as follows:

- Calculate Faber-Schauder wavelet transformation of the supposed marked image.

- Select the dominant blocks: blocks with high density of wavelet dominant coefficients.

- Calculate the correlation between the selected blocks and the random sequence $\mathbf{U}$ :

$$
\mathrm{r}(\mathrm{B}, \mathrm{U})=\frac{\sum_{m} \sum_{n}\left(B_{m n}-\bar{B}\right)\left(U_{m n}-\bar{U}\right)}{\sqrt{\left(\sum_{m} \sum_{n}\left(B_{m n}-\bar{B}\right)^{2}\right)\left(\sum_{m} \sum_{n}\left(U_{m n}-\bar{U}\right)^{2}\right)}}
$$

With $\mathbf{B}$ the constructed vector from a given block.

- Choose the maximum of the calculated correlations, noted $\mathbf{r}_{\max }$.

- If $\mathbf{r}_{\max }$ superior to a threshold $\mathrm{T}$ then the image is considered marked.

\section{Results and Conclusion}

\subsection{Visual's Deterioration}

The first tests done to measure the image deterioration (by the watermark) are the calculation of the PSNR (table1). We can notie that the deteriorations are very little visible for the marked image (lena 512) (Fig. 7). As long as the threshold is lower than 10, PSNR remains well above $30 \mathrm{~dB}$.

The performances of a marking algorithm are measured by the degree of resistance to classic attacks and to filtering, We will use for the proposed method the bench attacks CheckMark[13], The test bench is generated from one or several marked images, Then we will apply the algorithm of detection on each attacked image, one tried to detect the mark and we look if the detection succeeded or no,

This decision is made while defining a threshold of detection; the threshold is equal to $\mathbf{0 . 4 0}$. It is an empirically chosen from several tests.

Table 1. PSNR for marked images

\begin{tabular}{|c|r|r|}
\hline The watermark strength. & PNSR $(\mathbf{d b})$ & correlation \\
\hline 2 & 37.4035 & 0.437552 \\
\hline 7 & 32.8847 & 0.633063 \\
\hline 10 & 30.2074 & 0.743258 \\
\hline 15 & 27.3072 & 0.805537 \\
\hline 20 & 25.0252 & 0.809627 \\
\hline 30 & 21.7905 & 0.929487 \\
\hline
\end{tabular}




\subsection{Robust Against Compression}
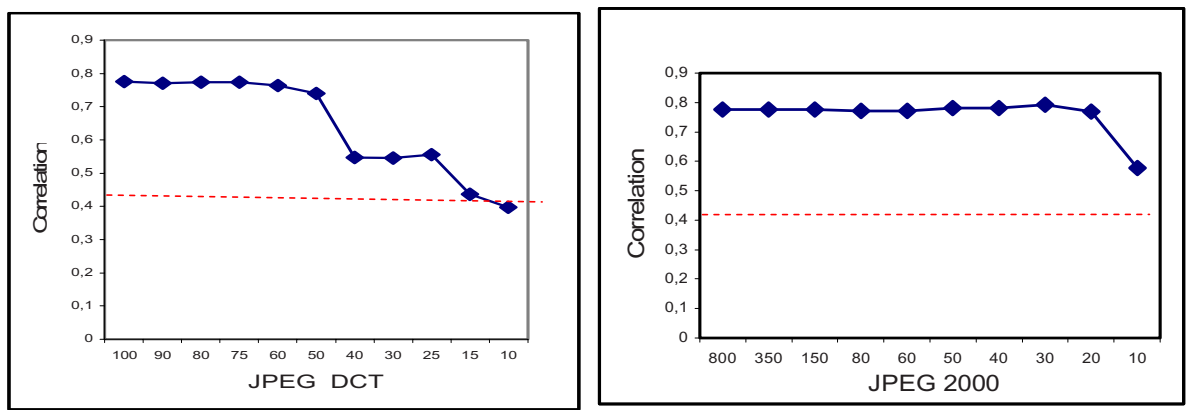

Fig. 3. The correlation for different qualities of Fig. 4. The correlation for different qualities compression by JPEG DCT: The resistance to of JPEG 2000 compression algorithm: it's the JPEG compression (DCT) is relatively always important (more far from the predeefficient fined threshold)

\subsection{Filtering}

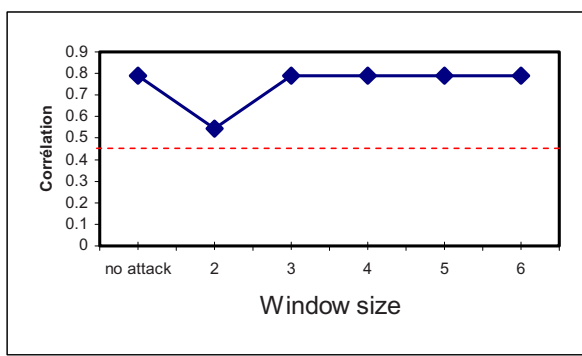

Fig. 5. The correlation obtained after an attack with the Gaussian filtering: the correlation still high than the threshold

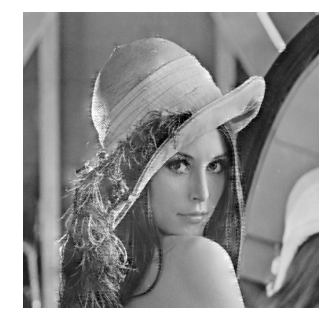

threshold $=20$, PNSR $=25.02 \mathrm{db}$

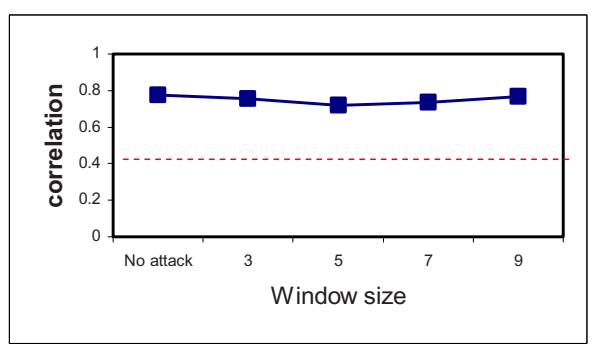

Fig. 6. The correlation obtained after an attack by the Wienner filtering: the correlation still very important

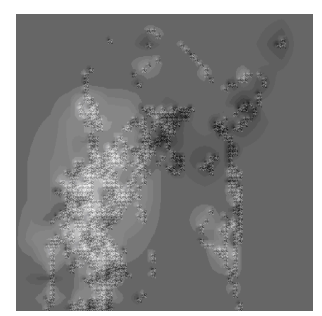

The difference image between the marked image and the original imag

Fig. 7. If the threshold of marking is too important, the distortions of the marked image are very important 


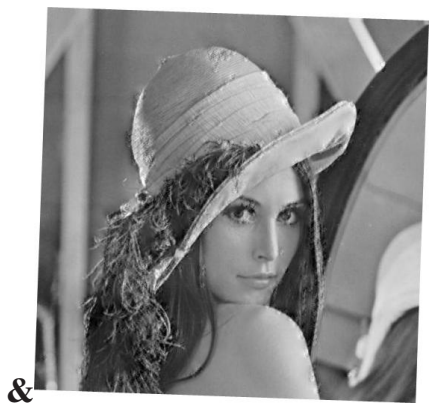

Example of the Rotation attack $\left(2^{\circ}\right)$ : correlation $=0.64>$ threshold

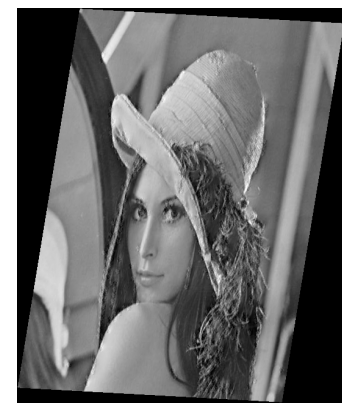

Example of cropping attack: correlation $=0.37<$ threshold

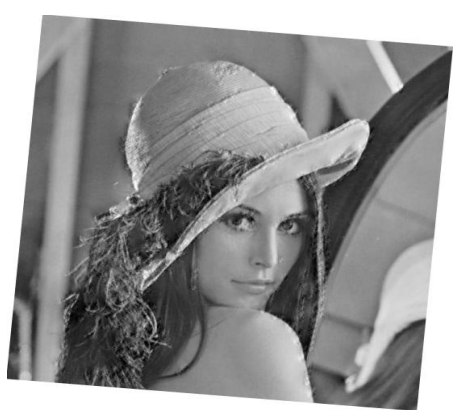

Example of the rotation attack $\left(4^{\circ}\right)$ : correlation $=0.41 \approx$ threshold

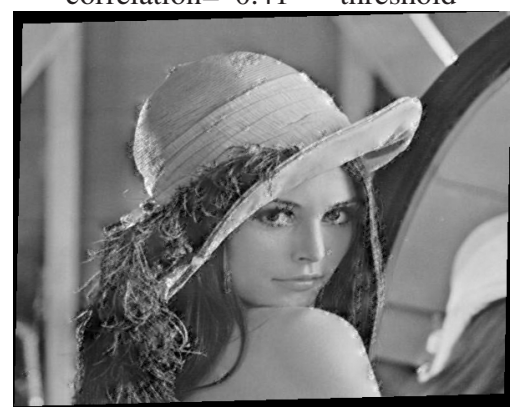

Example of cropping: correlation $=0.54>$ threshold

Fig. 8. Example of geometric attack

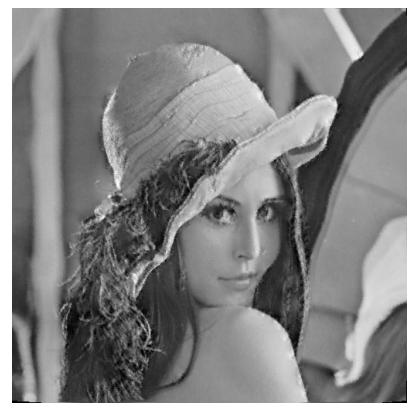

Fig. 9. Example of Stirmark attack: correlation $=0.42$

\subsection{Geometric Distortions}

The geometric attacks such as cropping, resizing and rotation attacks knock down the correlation scores significantly (cf. Fig 8 and 9). It is therefore necessary to improve the performances of this method, particularly by finding a solution to the problem of synchronization: Indeed the geometric distortion introduced a spatial desynchronization 
changing the density of blocks dominant of wavelet coefficients. So when we turn the image by $10^{\circ}$ the detection of the signature by the proposed method become ineffective. But for relatively small distortions (for example rotations lower to $4^{\circ}$ ) the detection of the signature still possible with our proposed method. The application that we aim has the particularity to provoke geometric distortions of very low amplitude [12] which shows that our approach could be successful.

\section{Conclusion}

In this papers we have presented a method of images watermarking based on mixedscales visualization by Faber-schauder wavelet. The experimental results have confirmed that this method is robust against various attacks: JPEG compression and geometric of small angle or displacement. It could therefore be used for images watermarking in this case. This method will be tested on images of access systems with magnetic badge where we have a geometric attack with small amplitude.

\section{References}

1. Petitcolas, A.P., Anderson, R.J., Kunh, M.G.: unformation hiding a survey. Proceedings of the IEEE 87, 1062-1078 (1999)

2. Cox, I., Miller, M.L.: A review of watermarking and the importance of perceptual modeling. In: Proceedings of SPIE, Humain visual and Electronic imaging II, vol. 3016, pp. 92 99 (1997)

3. Hartun, F., Kutter, M.: multimedia watermarking techniques. Proceedings of the IEEE 87, 1079-1107 (1999)

4. Cox, I., Killian, J., Leighton, F.T., Shamoon, T.: A secure robust watermark for multimedia. In: Anderson, R. (ed.) IH 1996. LNCS, vol. 1174, pp. 185-206. Springer, Heidelberg (1996)

5. Piva, A., Barni, M., Bartolini, F., Cappellini, V.: DCT-based watermark recovering without resorting to the uncorrupted original image. In: IEEE Signal Processing Society 1997 International Conference on Image Processing (ICIP 1997), Santa Barbara, California (1997)

6. Douzi, H., Mammass, D., Nouboud, F.: Faber-Schauder wavelet transformation application to edge detection and image characterization. Journal of Mathematical Imaging and Vision 14(2), 91-102 (2001)

7. Douzi, H., Mammass, D., Nouboud, F.: Amélioration de la compression par la transformation multiéchelles de Faber-Schauder. In: Vision Interface 1999 proceeding, 18-21 May, Trois-Rivières, Québec, Canada, pp. 424-431 (1999)

8. Cox, I.J., Kilian, J., Leighton, T., Shamoon, T.: Secure Spread Spectrum Watermarking for Multimedia. IEEE Trans. on Image Processing 6(12), 1673-1687 (1997)

9. Sweldens, W.: The lifting scheme: A construction of second generation wavelets. SIAM. J. Math. Anal. 29(2), 511-546 (1997)

10. Cox, I.J., Doërr, G., Furon, T.: Watermarking Is Not Cryptography. In: Shi, Y.Q., Jeon, B. (eds.) IWDW 2006. LNCS, vol. 4283, pp. 1-15. Springer, Heidelberg (2006)

11. Ros, F., Borla, J., Leclerc, F., Harba, R., Launay, N.: Watermarking for Plastic Card Supports. In: 9ème Conference Maghrébine sur Les Technologies de L'Information, MCSEAI, Agadir (2006) 
12. Chang, C., Maleki, A., Girod, B.: Adaptive Wavelet Transform for Image Compression via Directional Quincunx Lifting. In: Proc. IEEE International Workshop on Multimedia Signal Processing, MMSP 2005, Shanghai, China (2005)

13. Pereira, S., Voloshynovskiy, S., Madueño, M., Marchand-Maillet, S., Pun, T.: Second generation benchmarking and application oriented evaluation. In: Information Hiding Workshop III, Pittsburgh, PA, USA (April 2001)

14. Sweldens, W.: The lifting scheme: A construction of second generation wavelets. SIAM Journal on Mathematical Analysis 29(2), 511-546 (1998)

15. Kundur, D., Hatzinakos, D.: Digital Watermarking Using Multiresolution Wavelet Decomposition. In: Proc. IEEE Int. Conf. on Acoustics, Speech and Signal Processing, May 1998, vol. 5, pp. 2969-2972 (1998) 